\title{
Successful treatment of a locally advanced unresectable pancreatic cancer patient with interstitial pneumonitis by conversion surgery following gemcitabine plus nab-paclitaxel chemotherapy: A case report
}

\author{
TETSUO YOKOTA, SHIGETSUGU TAKANO, HIDEYUKI YOSHITOMI, SHINGO KAGAWA, \\ KATSUNORI FURUKAWA, TSUKASA TAKAYASHIKI, SATOSHI KUBOKI, DAISUKE SUZUKI, \\ NOZOMU SAKAI, HIROYUKI NOJIMA, TAKASHI MISHIMA, ERI NAKADAI and MASAYUKI OHTSUKA \\ Department of General Surgery, Graduate School of Medicine, Chiba University, Chiba 260-8677, Japan
}

Received December 11, 2018; Accepted January 30, 2019

DOI: $10.3892 /$ mco.2019.1807

\begin{abstract}
Conversion surgery is an attractive strategy to improve the outcomes for locally advanced unresectable (UR-LA) pancreatic ductal adenocarcinoma (PDAC). The present case report, presents a case of successful conversion surgery following the treatment of a patient with UR-LA PDAC, suffering from interstitial pneumonitis (IP), using a combination of gemcitabine and nab-paclitaxel (GnP). A 67-year-old woman presented at the hospital with a high level of carbohydrate antigen 19-9 (CA19-9; 1,713 U/ml). Radiological examination revealed a pancreatic tumor in contact with the superior mesenteric artery, with invasion extending to the most proximal draining jejunal branch into the superior mesenteric vein. The patient was diagnosed with UR-LA PDAC. Following 6 courses of GnP therapy, the tumor size markedly decreased from 50 to $18 \mathrm{~mm}$, and the level of CA19-9 also decreased from 1,713 to $60.1 \mathrm{U} / \mathrm{ml}$. Due to the progression of IP, the patient was administered steroid medication along with a restart of tacrolimus for the treatment of dermatomyositis and IP. After recovery from her lung condition, an additional 3 courses of GnP therapy
\end{abstract}

Correspondence to: Dr Shigetsugu Takano, Department of General Surgery, Graduate School of Medicine, Chiba University, 1-8-1 Inohana, Chuo-ku, Chiba 260-8677, Japan

E-mail: stakano@faculty.chiba-u.jp

Abbreviations: CA19-9, carbohydrate antigen 19-9; EUS, endoscopic ultrasonography; GS, gemcitabine plus S-1; GnP, gemcitabine plus nab-paclitaxel; IP, interstitial pneumonitis; MDCT, multi-detector row computed tomography; MST, median survival time; OS, overall survival; PET, positron emission tomography; PDAC, pancreatic ductal adenocarcinoma; PV, portal vein; SMA, superior mesenteric artery; SMV, superior mesenteric vein; UR-LA, locally advanced unresectable

Key words: conversion surgery, gemcitabine plus nab-paclitaxel, locally advanced unresectable pancreatic cancer, CA19-9, interstitial pneumonitis were administered, and then pancreatoduodenectomy was performed. The patient was still alive 14 months post-surgery with no recurrence. Between July 2009 and September 2017, conversion surgery was performed for 18 cases of UR-LA PDAC treated with gemcitabine plus S-1 (GS) therapy, and 11 cases with GnP therapy. The percentage of median CA19-9 and median tumor volume reductions were 73.7 and $51.6 \%$, respectively, following GS therapy, and 86.7 and $68.8 \%$, respectively, following $\mathrm{GnP}$ therapy. Tumor reduction following $\mathrm{GnP}$ therapy was significantly higher than that after GS therapy $(\mathrm{P}=0.02)$. $\mathrm{GnP}$ therapy is a suitable regimen to shrink the tumor mass in patients with UR-LA PDAC. Careful management of systemic conditions is required to treat patients with PDAC and IP when using GnP therapy. Conversion surgery should be considered for recognizing radiological responses (tumor shrinkage adjacent to major arteries) and reductions in CA19-9 levels.

\section{Introduction}

Pancreatic ductal adenocarcinoma (PDAC) is the fourth leading cause of cancer-related deaths worldwide with an unfavorable 5-year survival rate of $8 \%$ (1). National Comprehensive Cancer Network (NCCN) recommends chemotherapy or chemo-radiation therapy for the treatment of locally advanced unresectable (UR-LA) PDAC. However, these therapies do little to improve disease prognosis. Recently, surgery after chemo-radiation therapy, i.e., conversion surgery, for UR PDAC was reported to improve prognosis (2). We demonstrated a case of successful conversion surgery following treatment of an UR-LA PDAC patient with interstitial pneumonitis using a gemcitabine and nab-paclitaxel $(\mathrm{GnP})$ therapy. In addition, we retrospectively compare clinical efficacy of two chemotherapeutic regimens, GnP therapy and gemcitabine plus S-1 (GS) therapy for treatment of UR-LA PDAC.

\section{Case report}

A 67-year-old woman suffering from dermatomyositis and interstitial pneumonitis (IP) controlled by an immunosuppressive 
agent, tacrolimus, was referred to the Department of General Surgery, Chiba University Hospital. Laboratory data reported a high level of carbohydrate antigen 19-9 (CA19-9; $1,713 \mathrm{U} / \mathrm{ml}$ ), with no other remarkable laboratory findings. Abdominal multi-detector row computed tomography (MDCT) revealed a hypovascular tumor measuring $50 \mathrm{~mm}$ in the head of the pancreas. The tumor was in contact with the superior mesenteric artery (SMA), with invasion extending to the most proximal draining jejunal branch into the superior mesenteric vein (SMV) (Fig. 1A and B). Further, the tumor spread over a third of the duodenum. Endoscopic ultrasonography (EUS) also indicated that the tumor was in contact with both the SMA and the SMV, and fine needle aspiration biopsy revealed adenocarcinoma. Positron emission tomography (PET) exhibited fluorodeoxyglucose uptake in the primary pancreatic tumor (Fig. 1C); however, both PET and ethoxybenzyl-magnetic resonance imaging showed no evidence of distant metastasis.

On the basis of these clinical findings, the patient was diagnosed with UR-LA PDAC. She understood the risk of exacerbating her IP, and agreed to treatment with a combined regimen of gemcitabine (GEM, 1,000 $\mathrm{mg} / \mathrm{m}^{2}$ ) and nab-paclitaxel $\left(125 \mathrm{mg} / \mathrm{m}^{2}\right)$, with subsequent conversion surgery. GnP chemotherapy was administered intravenously on days 1 and 8 and was repeated every three weeks. Before the start of GnP therapy, we consulted a physician specializing in allergy and collagen diseases and stopped administering tacrolimus. After six courses of GnP administration over five months, MDCT, EUS, and PET imaging demonstrated a significant response to chemotherapy (Fig. 1D-F). Tumor size decreased to 18 $\mathrm{mm}$, contact with the SMV was reduced from 180 degrees to 90 degrees and the SMA separated from the tumor. The level of CA19-9 decreased from 1,713 to $60.1 \mathrm{U} / \mathrm{ml}$ (Fig. 2B). Despite these positive clinical responses, we could not schedule conversion surgery because the patient's IP had worsened (Fig. 2A). For one month after steroid medication and restart of regular tacrolimus administration, lung function recovered. Despite the lack of tumor growth on MDCT, she was treated with three additional courses of $\mathrm{GnP}$ chemotherapy over two months because CA19-9 level had increased to $132.1 \mathrm{U} / \mathrm{ml}$. After the additional chemotherapy, CA19-9 level decreased to 99.5 U/ml, and CT and EUS showed a partial response (PR) to chemotherapy using RECIST criteria with controlled IP. Based on criteria from the UICC-the 8th edition, TNM staging of before and after chemotherapy were T4NOM0 stage III and T1cN0M0 stage IA, respectively. After discussion with the patient and her family, conversion surgery was planned.

Pancreaticoduodenectomy was performed. Intraoperative pathological examination of a frozen section showed that the margins of the bile duct and stump of the pancreas were negative for cancer tissue (Fig. 3A). Histological examination showed R0 (no residual tumor) resection, and less than $10 \%$ of tumor cells were replaced with fibrosis (Evans' criteria I; Fig. 3B). From pathological findings (well differentiated tubular adenocarcinoma, pT2 (20x15 mm), positive for common bile duct, duodenum, and perineural invasion, pN0 (0/20), pM0), the tumor was defined as f-stage IB. Although the patient suffered from chylous ascites after surgery, the patient made a satisfactory recovery and was discharged on postoperative day 42. After administration of adjuvant chemotherapy (S-1, $100 \mathrm{mg} /$ day) for 6 months, the patient is vigorously alive at
23 months after initial treatment (14 months after surgery), with no recurrence.

Accumulative rates for the duration of chemotherapy prior to surgery were calculated using the Kaplan-Meier method. Statistical significance of the results was determined by Mann-Whitney $\mathrm{U}$ test. $\mathrm{P}<0.05$ was considered to indicate a statistically significant difference. Statistical calculations were performed using the $\mathrm{JMP}^{\circledR} 13$ (SAS Institute, Inc., Cary, NC, USA).

\section{Discussion}

Surgical resection offers the only chance for cure in patients with PDAC. Conversion surgery with multidisciplinary therapy is an attractive and crucial treatment for UR-LA PDAC. Satoi et al (3) reported that conversion surgery for UR-LA significantly prolonged survival compared to survival without conversion surgery. Median overall survival (OS) time (MST) was 39.7 and 20.8 months following conversion surgery compared with a control group (3). Asano et al (4) reported that MST was 3.8 years in the conversion surgery group. Recently, Okura et al (5) reported that Kaplan-Meier analysis showed that patients treated with GnP therapy followed by conversion surgery presented significantly longer OS than those treated with GnP therapy without conversion surgery (MST: 22.5 vs. 11 months) (5).

The MPACT study demonstrated that GnP therapy is effective for treatment of metastatic PDAC (6). This therapy draws attention as standard chemotherapy for UR PDAC. Saito et al (7) showed that, after GnP therapy for UR-LA, tumor reduction was $37 \%$, response rate was $71 \%$, and conversion rate was $29 \%$. Another chemotherapeutic regimen used to treat UR PDAC, FOLFIRINOX (5-Fluorouracil/leucovorin combined with irinotecan and oxaliplatin), is also used for chemotherapy preceding conversion surgery (8). Giovanni showed that response rate was $49 \%$ and conversion rate was $72.9 \%$ for UR-LA and borderline resectable PDAC (9). Furthermore, the percentage of UR-LA PDAC patients was reduced from 78 to $32 \%$ by use of FOLFIRINOX. Muranaka et al (10) have described that GnP (40.9\%) showed higher response rate than that of FOLFIRINOX $(6.3 \%)$ in UR PDAC; however, the superiority of including conversion surgery with either of these two regimes is still under debate. GS therapy is also a useful PDAC treatment, especially for Asian patients. The GEST study reported that response rates for GEM, S-1, and GS therapies were 12.9, 21.0 and 28.9\%, respectively with a low incidence of adverse side effects (11). Thus, GS therapy is also considered an appropriate chemotherapeutic regimen for UR PDAC.

CA19-9 level is a reasonably reliable indicator of whether conversion surgery should be considered. Previous studies show that CA19-9 response to neoadjuvant therapy is closely related to high $\mathrm{R} 0$ resection rate and prolongation of survival $(12,13)$. In the present case, we decided to continue GnP treatment until CA19-9 level dropped because CA19-9 level in the patient increased slighted after steroid medication despite lack of progression of the tumor in radiological imaging.

A total of 29 cases treated with conversion surgery after chemotherapy for UR-LA PDAC between July 2009 and 

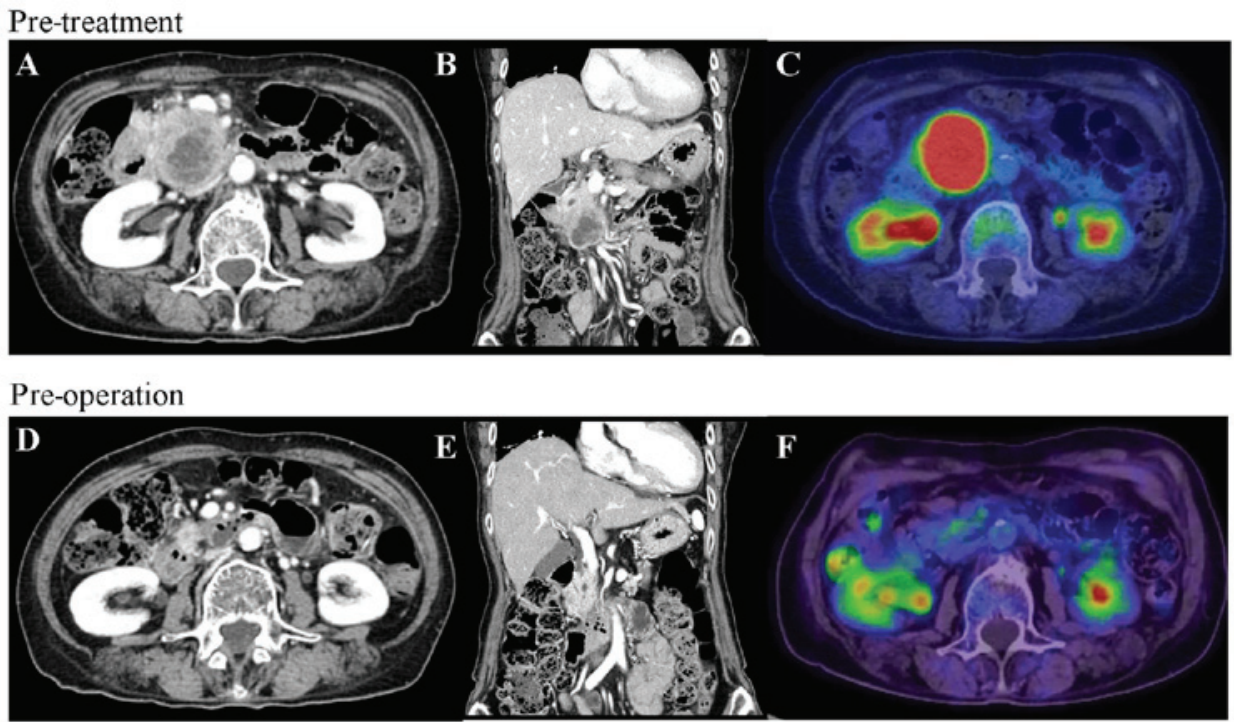

Figure 1. CT and PET before and after gemcitabine plus nab-paclitaxel therapy. (A) CT at the initial visit showed a hypovascular tumor measuring $50 \mathrm{~mm}$ in the head of the pancreas. The tumor was in contact with the SMA. (B) Tumor invasion extended to the most proximal draining jejunal branch into SMV and the tumor spread over one third of the duodenum. (C) PET at the initial visit showed high fluorodeoxyglucose uptake into the primary pancreatic tumor (D) Tumor size decreased to $18 \mathrm{~mm}$ post-operation, contact with the SMV decreased to 90 degrees and the tumor separated from the SMA. (E) Tumor invasion in proximal draining jejunal branch into the SMV disappeared. (F) Fluorodeoxyglucose uptake into the primary pancreas tumor was eliminated. CT, computed tomography; PET, positron emission tomography; SMA, superior mesenteric artery; SMV, superior mesenteric vein.

A

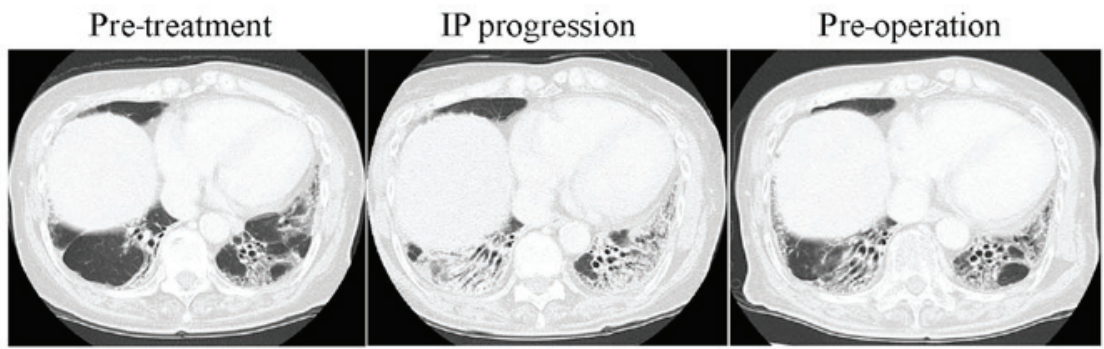

B

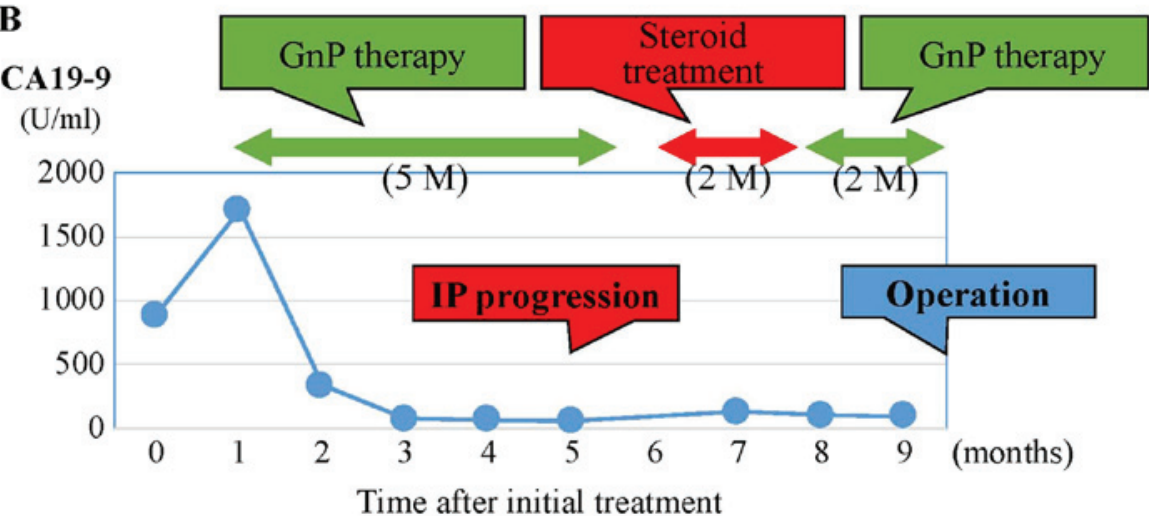

Figure 2. CT images of the lung field and changes in serum CA19-9 levels during chemotherapy and prior to surgery. (A) Lung images prior to the initial treatment (left panel), after IP progression (middle panel), and improvements following steroid treatment (right panel). (B) The change in serum CA19-9 levels during the course of chemotherapy. CA19-9 levels were reduced markedly following 5 months of GnP therapy. At 2 months post-steroid treatment, GnP was therapy re-administered for 2 months. CT, computed tomography; CA19-9, carbohydrate antigen 19-9; IP, interstitial pneumonitis; GnP, gemcitabine plus nab-paclitaxel.

September 2017 (18 cases: GS therapy and 11 cases: GnP therapy) were identified (according to the NCCN guideline 2018.2) from records from the Department of General Surgery, Chiba University Hospital. In general, the indication for conversion surgery of these cases were determined on the basis of the significant reduction of serum CA19-9 level and loss of arterial invasion of tumor in MDCT imaging. The median duration of chemotherapy followed by surgery was 3.1 months after GS therapy, and 4.4 months after GnP therapy (Fig. 4A). To compare chemotherapeutic efficacy, median reduction rates in CA19-9 levels were 86.7 and $73.7 \%$ after GnP and GS therapy, respectively (Fig. 4B). 
A

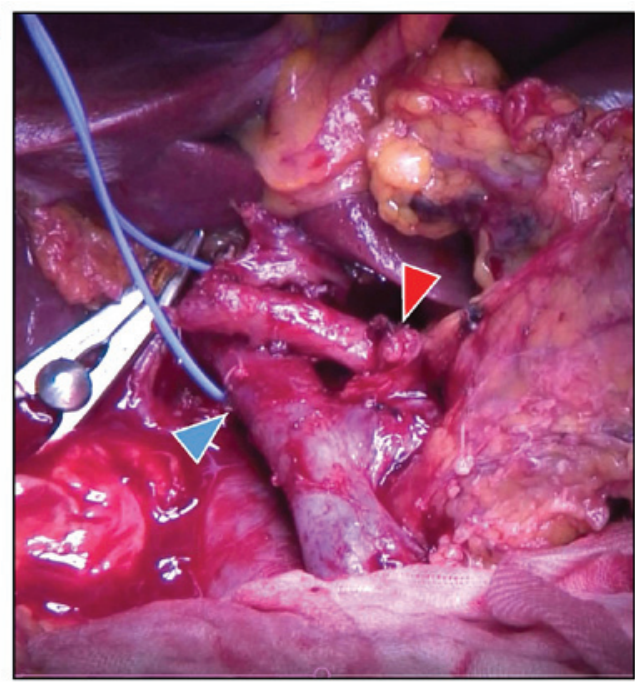

B

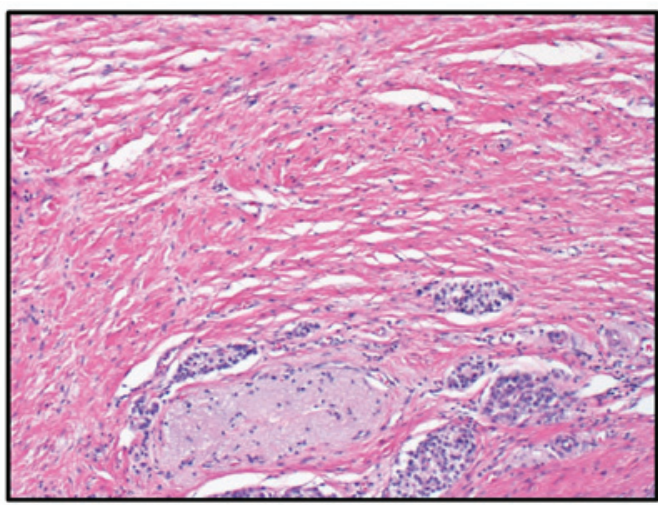

Figure 3. Intraoperative and pathological images. (A) Intraoperative image. Gastroduodenal artery stump is indicated by the red arrowhead and the portal vein is indicated by the blue arrowhead. (B) Microscopic findings, following chemotherapy, of the surgical specimen showing a change of $<10 \%$ in the fibrous tissue with grade I on Evans' grade criteria. Staining was performed with hematoxylin and eosin (magnification, x40).
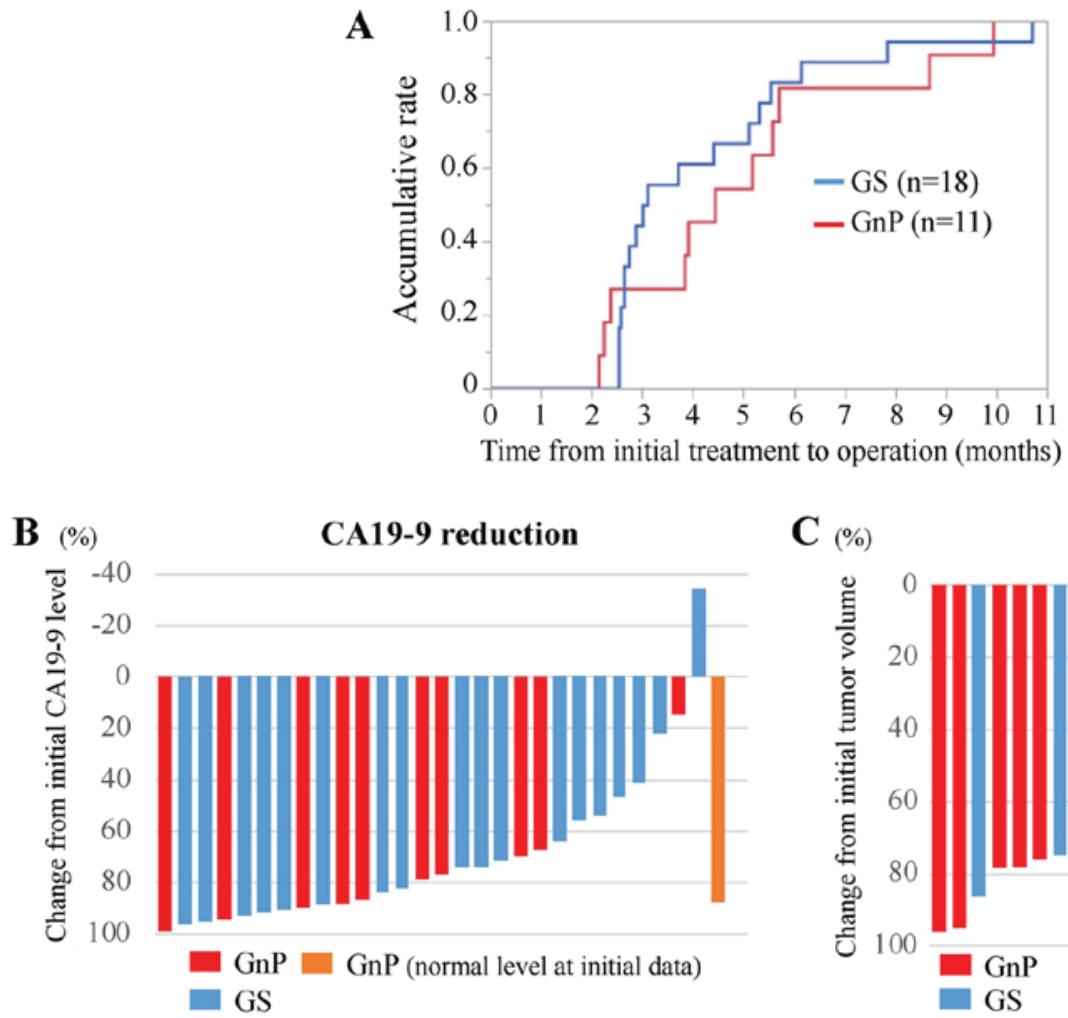

$\mathbf{C}(\%)$

Tumor shrinkage

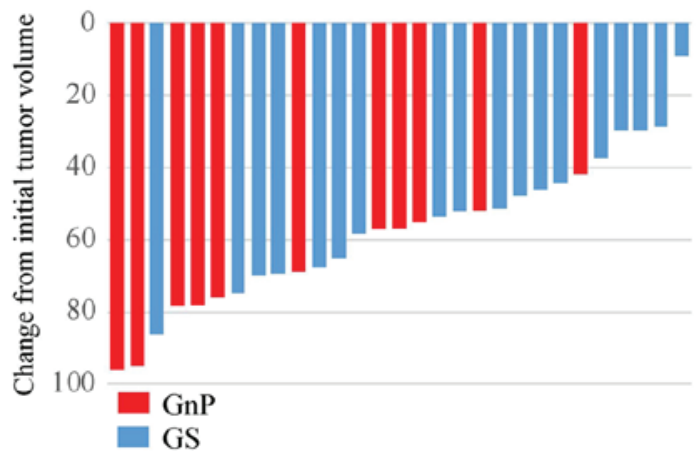

Figure 4. Comparative analysis of clinical parameters between GnP and GS therapy. (A) Comparative analysis of time from initial treatment to operation for the GnP (red line) and GS (blue line) therapy groups. (B) Waterfall plot analysis for CA19-9 reduction from initial treatment to operation during GnP (red and orange bars) and GS (blue bars) therapy. The orange bar indicates a case where their serum CA19-9 level at initial treatment was within normal range. (C) Waterfall plot analysis for tumor shrinkage from initial treatment to operation for GnP (red bars) and GS (blue bars) therapy. Tumor volume was calculated using the following formula: $\pi /[6 x(\mathrm{LxWxW})]$, where $\mathrm{L}$ is the tumor length and $\mathrm{W}$ is the width. CA19-9, carbohydrate antigen 19-9; GnP, gemcitabine plus nab-paclitaxel; GS, gemcitabine plus S-1.

As shown in the present case, GnP therapy showed a greater reduction of tumor volume. Tumor reduction rates were significantly higher following GnP than after GS therapy, medians: 68.8 and $51.6 \%$, respectively in this retrospective cohort ( $\mathrm{P}=0.02$, Mann-Whitney Wilcoxon test) (Fig. 4C). The mechanism for this difference may involve stroma reduction induced by GnP therapy (14). GnP therapy may be suitable for conversion surgery because of this additional biological effect to the tumor volume reduction.

Chemotherapy causes a variety of adverse side-effects. In the present case, the patient had progressive IP during GnP therapy. Studies have shown that severe interstitial lung disease 
(ILD) has occurred following gemcitabine-based chemotherapy $(15,16)$. These studies reported an occurrence rate from 1.7 to $7.6 \%$, and risk factors were prior thoracic radiotherapy, pre-existing pulmonary fibrosis, age of over 80 years, and lung cancer. The occurrence rate of ILD induced by nab-paclitaxel is reported as $6 \%$ (17). In this case, FOLFIRINOX was not selected because reported adverse events were more frequent in Japanese patients when compared with GnP therapy (18). IP did progress after GnP therapy; however cessation of immunosuppressive treatment for dermatomyositis and IP may be the underlying factor. Thus, collaboration between medical specialists for allergy and oncology is necessary to prevent progression of IP during GnP treatment for PDAC patients with ILD.

In conclusion, we describe a case of successful conversion surgery following gemcitabine plus nab-paclitaxel treatment of UR-LA PDAC. GnP therapy followed by conversion surgery is a suitable regimen to shrink UR PDAC tumors; however, long-term clinical outcomes need further investigation. Of particular note is that careful management of systemic conditions is needed to treat PDAC patients with IP using GnP therapy. Conversion surgery should be considered recognizing radiological responses (tumor shrinkage adjacent to major arteries) and reductions in CA19-9 levels. Further evidence and prospective cohort studies are necessary to establish an optimal strategy for treatment of UR-LA PDAC.

\section{Acknowledgements}

Not applicable.

\section{Funding}

The present study was supported by the Grant-in-Aid for Scientific Research, The Challenge Exploratory Research (grant no. 16K15607). This study was also supported by Kashiwado Memorial Foundation and Japanese Foundation for Multidisciplinary Treatment of Cancer.

\section{Availability of data and materials}

The datasets used and/or analyzed during the current study are available from the corresponding author on reasonable request.

\section{Authors' contributions}

TY obtained the patient's data and wrote the manuscript. ST treated the patient with preoperative and adjuvant chemotherapy. MO and ST performed the surgery. TM and MO were responsible for the pathological diagnosis of the case. HY, KF, TT, SaK, DS, NS, ShK, HN, EN, and MO discussed, analyzed and interpreted the data with TY and ST, and assisted in writing the manuscript. All authors approved the final manuscript.

\section{Ethics approval and consent to participate}

The Ethics Committees of Chiba University approved the content of this manuscript (\#2732), and written informed consent was obtained from each patient prior to surgery.

\section{Patient consent for publication}

Written informed consent was obtained from the patient for publication of this case report and any accompanying images.

\section{Competing interests}

The authors declare that they have no competing interests.

\section{References}

1. Siegel RL, Miller KD and Jemal A: Cancer statistics, 2018. CA Cancer J Clin 68: 7-30, 2018.

2. Tempero MA, Malafa MP, Al-Hawary M, Asbun H, Bain A, Behrman SW, Benson AB III, Binder E, Cardin DB, Cha C, et al; National Comprehensive Cancer Network Clinical Practice Guidelines in Oncology: Pancreatic Adenocarcinoma, Version 2.2017, NCCN Clinical Practice Guidelines in Oncology. J Natl Compr Canc Netw 15: 1028-1061, 2017.

3. Satoi S, Yamaue H, Kato K, Takahashi S, Hirono S, Takeda S, Eguchi H, Sho M, Wada K, Shinchi $\mathrm{H}$, et al: Role of adjuvant surgery for patients with initially unresectable pancreatic cancer with a long-term favorable response to non-surgical anti-cancer treatments: Results of a project study for pancreatic surgery by the Japanese Society of Hepato-Biliary-Pancreatic Surgery. J Hepatobiliary Pancreat Sci 20: 590-600, 2013.

4. Asano T, Hirano S, Nakamura T, Okamura K, Tsuchikawa T, Noji T, Nakanishi Y, Tanaka K and Shichinohe T: Survival benefit of conversion surgery for patients with initially unresectable pancreatic cancer who responded favorably to nonsurgical treatment. J Hepatobiliary Pancreat Sci 25: 342-350, 2018.

5. Okura R, Takano S, Yokota T, Yoshitomi H, Kagawa S, Furukawa K, Takayashiki T, Kuboki S, Suzuki D, Sakai N, et al: Conversion surgery with gemcitabine plus nab-paclitaxel for locally advanced unresectable pancreatic cancer: A case report. Mol Clin Oncol 9: 389-393, 2018.

6. Von Hoff DD, Ervin T, Arena FP, Chiorean EG, Infante J, Moore M, Seay T, Tjulandin SA, Ma WW, Saleh MN, et al: Increased survival in pancreatic cancer with nab-paclitaxel plus gemcitabine. N Engl J Med 369: 1691-1703, 2013.

7. Saito T, Ishido K, Kudo D, Kimura N, Wakiya T, Nakayama Y and Hakamada K: Combination therapy with gemcitabine and nab-paclitaxel for locally advanced unresectable pancreatic cancer. Mol Clin Oncol 6: 963-967, 2017.

8. Blazer M, Wu C, Goldberg RM, Phillips G, Schmidt C, Muscarella P, Wuthrick E, Williams TM, Reardon J, Ellison EC, et al: Neoadjuvant modified (m) FOLFIRINOX for locally advanced unresectable (LAPC) and borderline resectable (BRPC) adenocarcinoma of the pancreas. Ann Surg Oncol 22: 1153-1159, 2015

9. Marchegiani G, Todaro V, Boninsegna E, Negrelli R, Sureka B Bonamini D, Salvia R, Manfredi R, Pozzi Mucelli R and Bassi C: Surgery after FOLFIRINOX treatment for locally advanced and borderline resectable pancreatic cancer: Increase in tumour attenuation on CT correlates with R0 resection. Eur Radiol 28: 4265-4273, 2018.

10. Muranaka T, Kuwatani M, Komatsu Y, Sawada K, Nakatsumi H, Kawamoto Y, Yuki S, Kubota Y, Kubo K, Kawahata S, et al: Comparison of efficacy and toxicity of FOLFIRINOX and gemcitabine with nab-paclitaxel in unresectable pancreatic cancer. J Gastrointest Oncol 8: 566-571, 2017.

11. Ueno H, Ioka T, Ikeda M, Ohkawa S, Yanagimoto H, Boku N, Fukutomi A, Sugimori K, Baba H, Yamao K, et al: Randomized phase III study of gemcitabine plus S-1, S-1 alone, or gemcitabine alone in patients with locally advanced and metastatic pancreatic cancer in Japan and Taiwan: GEST study. J Clin Oncol 31: 1640-1648, 2013.

12. van Veldhuisen E, Vogel JA, Klompmaker S, Busch OR, van Laarhoven HWM, van Lienden KP, Wilmink JW, Marsman HA and Besselink MG: Added value of CA19-9 response in predicting resectability of locally advanced pancreatic cancer following induction chemotherapy. HPB (Oxford) 20: 605-611, 2018.

13. Boone BA, Steve J, Zenati MS, Hogg ME, Singhi AD, Bartlett DL, Zureikat AH, Bahary N and Zeh HJ III: Serum CA 19-9 response to neoadjuvant therapy is associated with outcome in pancreatic adenocarcinoma. Ann Surg Oncol 21: 4351-4358, 2014. 
14. Alvarez R, Musteanu M, Garcia-Garcia E, Lopez-Casas PP, Megias D, Guerra C, Muñoz M, Quijano Y, Cubillo A, Rodriguez-Pascual J, et al: Stromal disrupting effects of nab-paclitaxel in pancreatic cancer. Br J Cancer 109: 926-933, 2013.

15. Hamada T, Yasunaga $H$, Nakai Y, Isayama $H$, Matsui $H$, Fushimi K and Koike K: Interstitial lung disease associated with gemcitabine: A Japanese retrospective cohort study. Respirology 21: 338-343, 2016

16. Umemura S, Yamane H, Suwaki T, Katoh T, Yano T, Shiote Y, Takigawa N, Kiura K and Kamei H: Interstitial lung disease associated with gemcitabine treatment in patients with non-small-cell lung cancer and pancreatic cancer. J Cancer Res Clin Oncol 137: $1469-1475,2011$
17. Nakaya A, Kurata T, Yokoi T, Takeyasu Y, Niki M, Kibata K, Satsutani N, Torii Y, Katashiba Y, Ogata M, et al: Retrospective analysis of single-agent nab-paclitaxel in patients with platinum-resistant non-small cell lung cancer. Mol Clin Oncol 7: 803-807, 2017.

18. Okusaka T, Ikeda M, Fukutomi A, Ioka T, Furuse J, Ohkawa S, Isayama $\mathrm{H}$ and Boku N: Phase II study of FOLFIRINOX for chemotherapy-naïve Japanese patients with metastatic pancreatic cancer. Cancer Sci 105: 1321-1326, 2014.

(i) $\Theta$ This work is licensed under a Creative Common Attribution-NonCommercial-NoDerivatives 4.0 International (CC BY-NC-ND 4.0) License. 